\title{
Janus nanosheets synchronously strengthen and toughen polymer blends
}

Yu Hou ${ }^{1,2}$, Guolin Zhang ${ }^{1}$, Xiuping Tang ${ }^{1}$, Yan $\mathrm{Si}^{2}$, Xi-Ming Song ${ }^{1}$, Fuxin Liang*1,2, Zhenzhong Yang*2,3

1. Liaoning Provincial Key Laboratory for Green Synthesis and Preparative Chemistry of Advanced Materials, Liaoning University, Shenyang 110036, China.

2. State Key Laboratory of Polymer Physics and Chemistry, Institute of Chemistry, Chinese Academy of Sciences, Beijing 100190, China.

3. Institute of Polymer Science and Engineering, Department of Chemical Engineering, Tsinghua University, Beijing 100084, China.

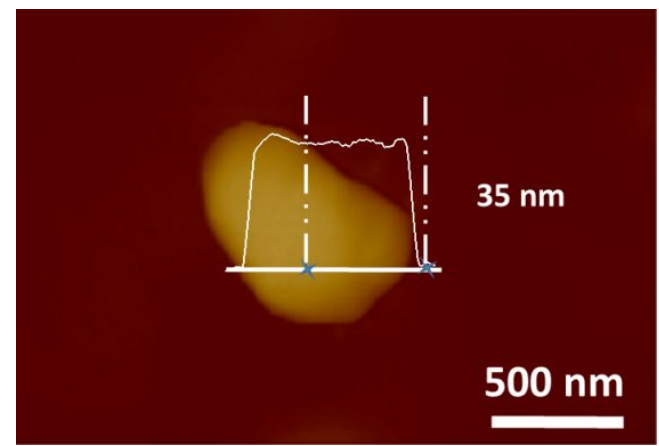

Figure S1. AFM image of the silica JN.

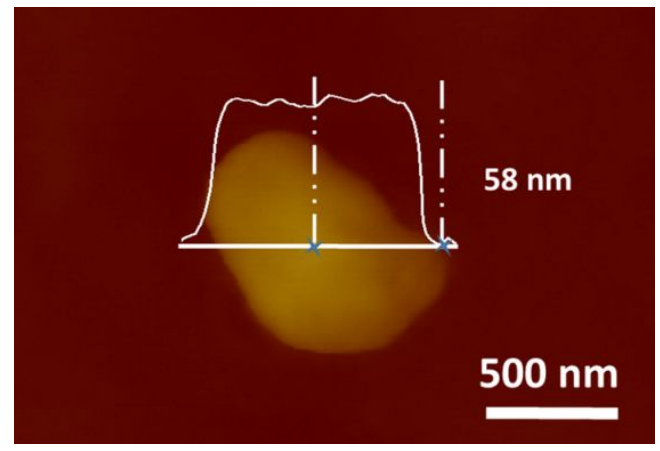

Figure S2. AFM image of the NBR JN. 


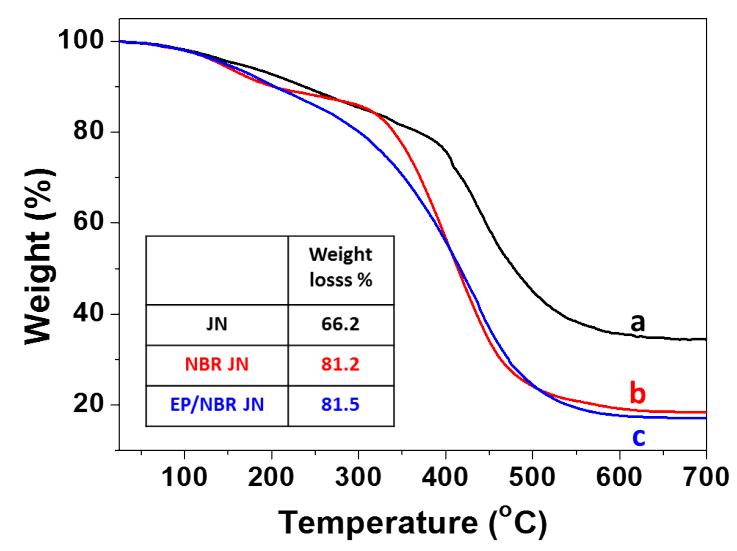

Figure S3. TGA curves of a) the silica JN; b) the NBR JN; c) the EP/NBR JN

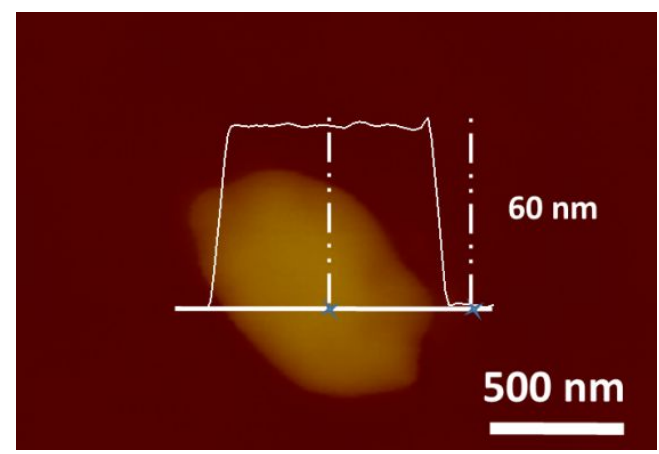

Figure S4. AFM image of the EP/NBR JN.

a)
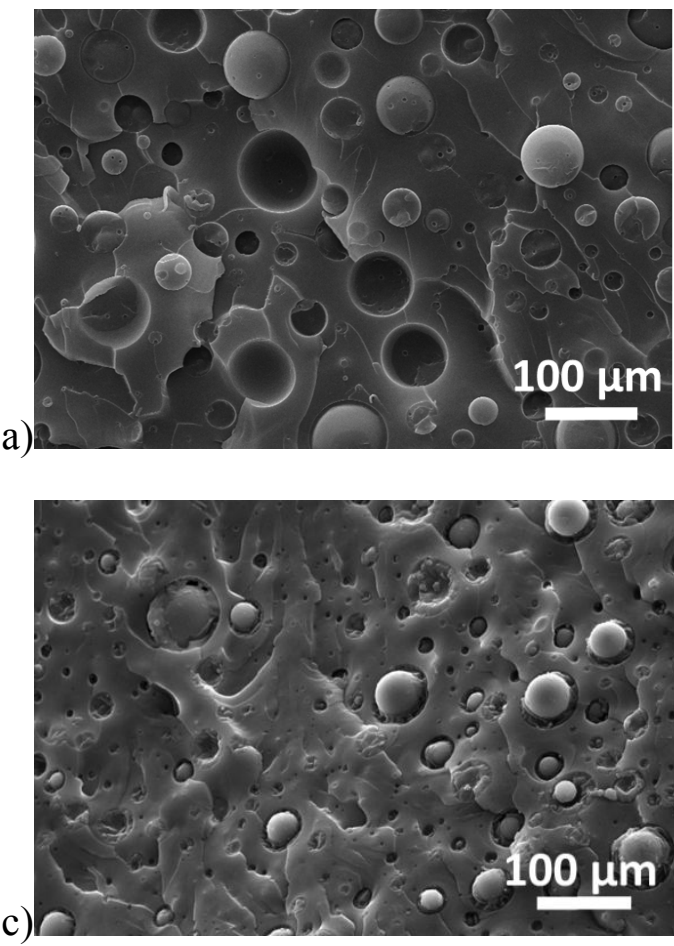

b)
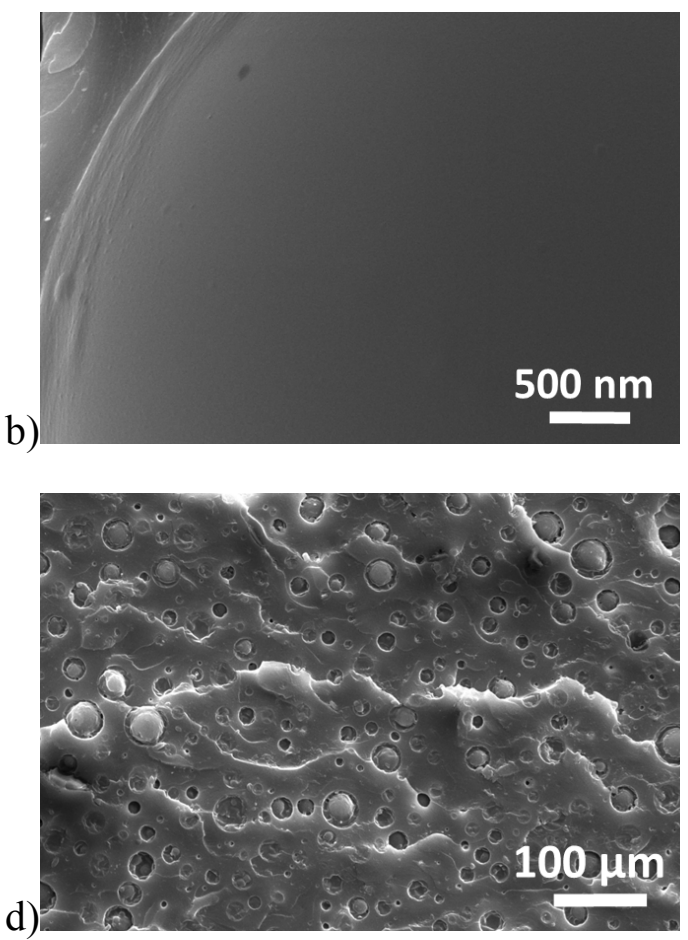

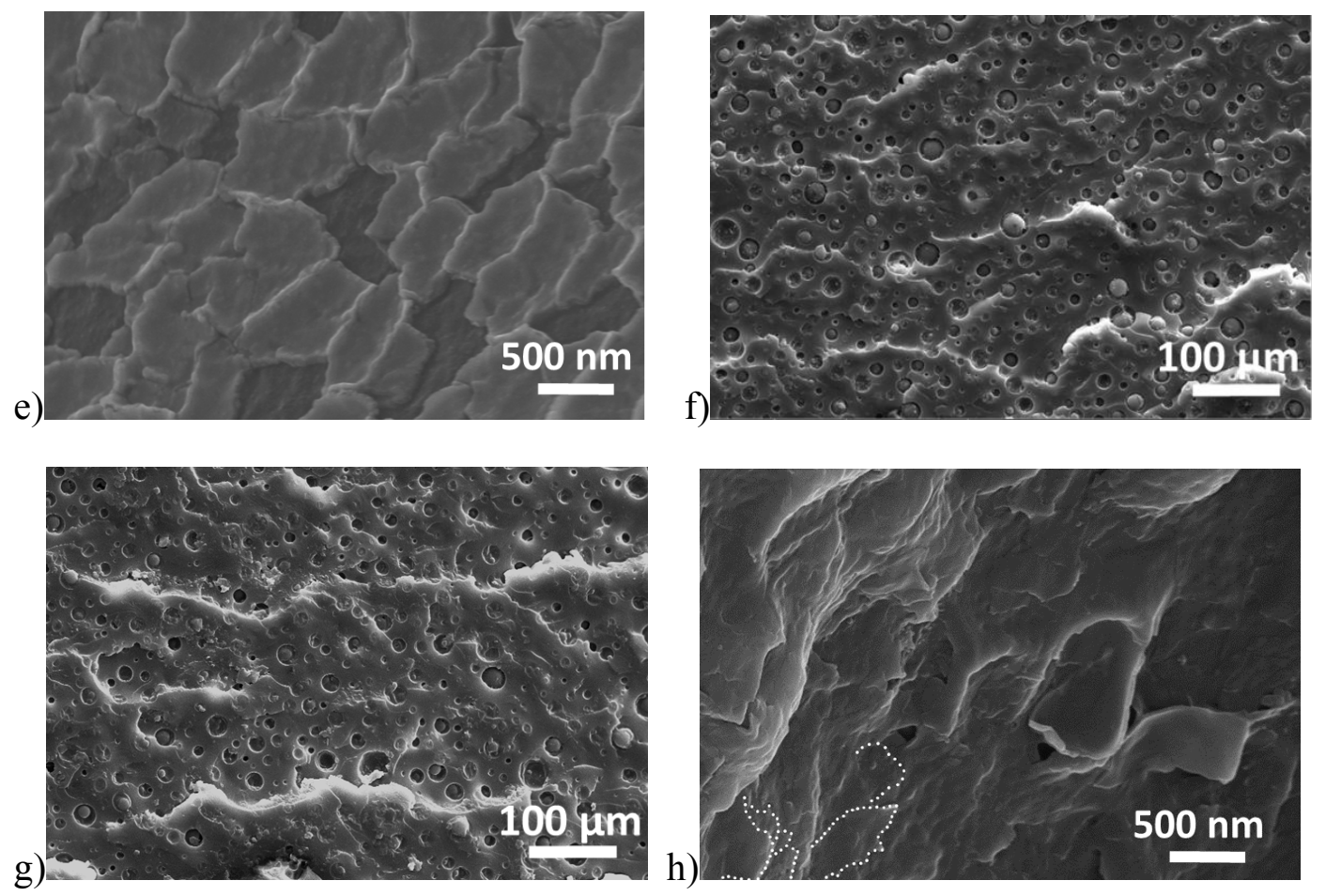

Figure S5. a, b) SEM and the magnified images of the fractured surface of the EP/10-LNBR blend; SEM images of fractured surfaces of the EP/10-LNBR blends at varied content of the JN (phr): c) $0.02 ;$ d) 0.14 and e) the magnified SEM image of the cavity; f) 0.20 ; g) 0.40 and h) the magnified SEM image of the cavity.

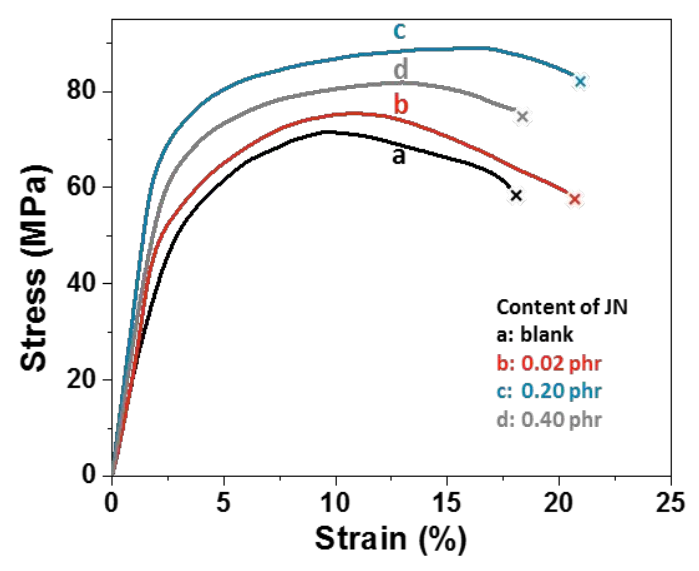

Figure S6. Tensile curves of the EP/10-LNBR blend a) and after feeding varied content of the EP/NBR JN (phr): b) 0.02 ; c) 0.20 ; d) 0.40 . 


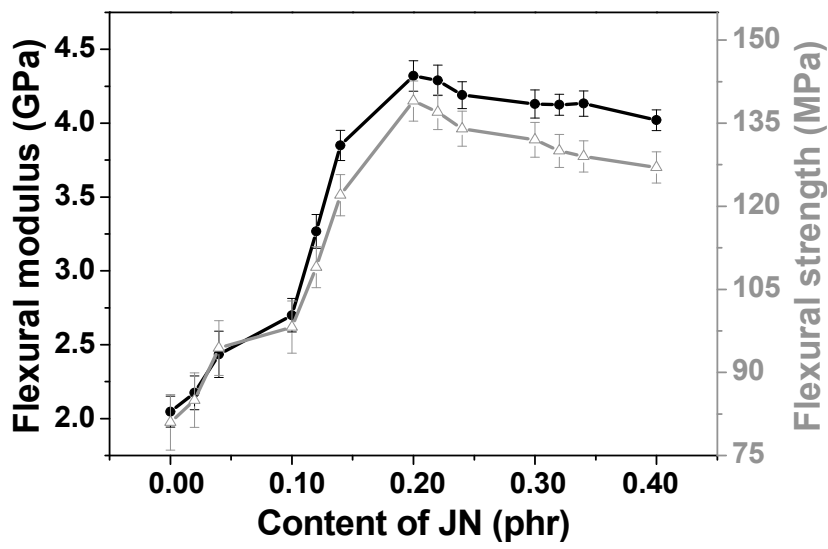

Figure S7. Flexural modulus $(\bullet)$ and strength (grey $\Delta$ ) of the EP/10-LNBR blend as a function of the content of the EP/NBR JN.

a)

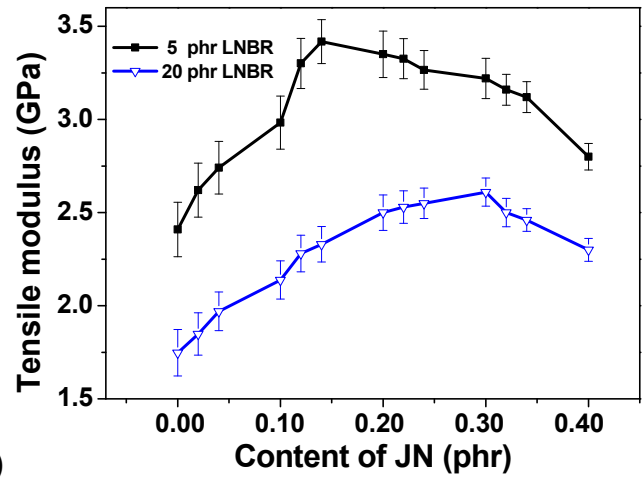

c)
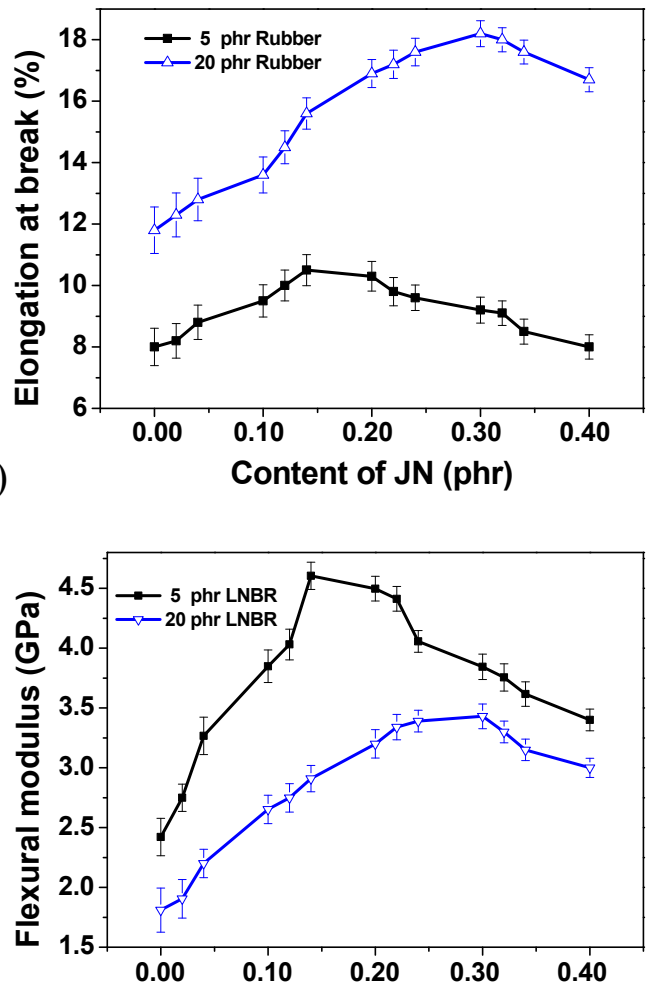

e)

Content of JN (phr)

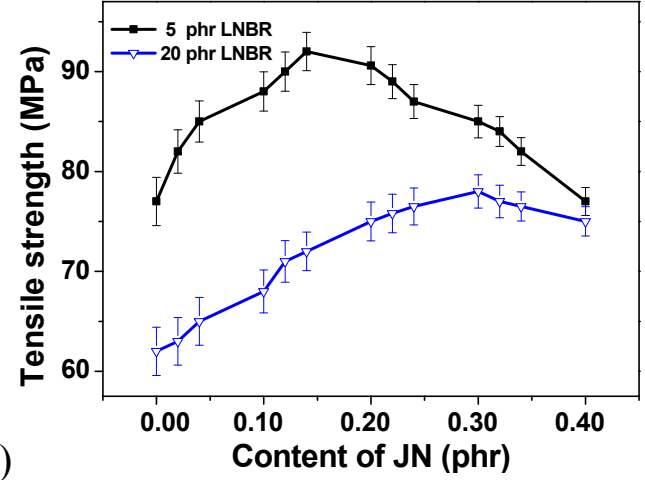

b)

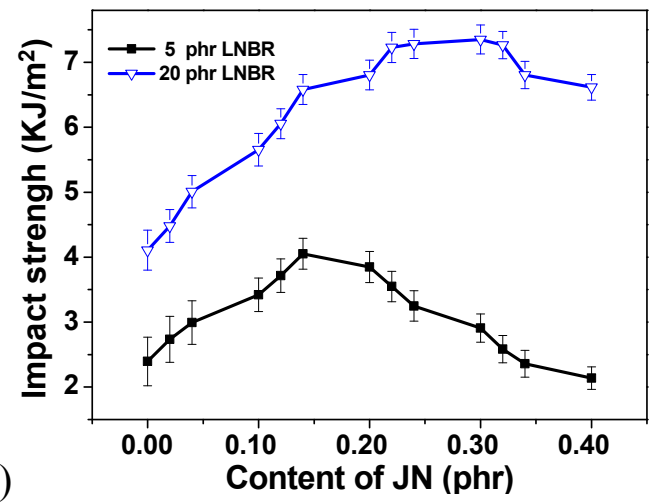

d)

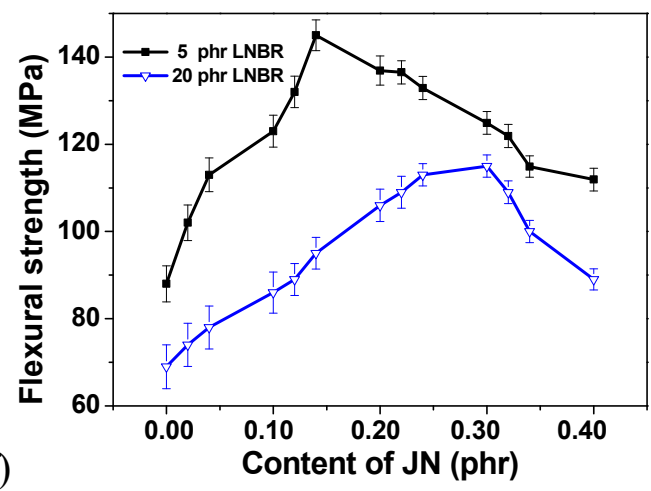


Figure S8. Mechanical properties of the EP/5-LNBR blend (a) and the EP/20-LNBR blend $(\nabla)$ as a function of the EP/NBR JN content: a) tensile modulus; b) tensile strength; c) elongation at break; d) impact strength; e) flexural modulus; f) flexural strength.

a)

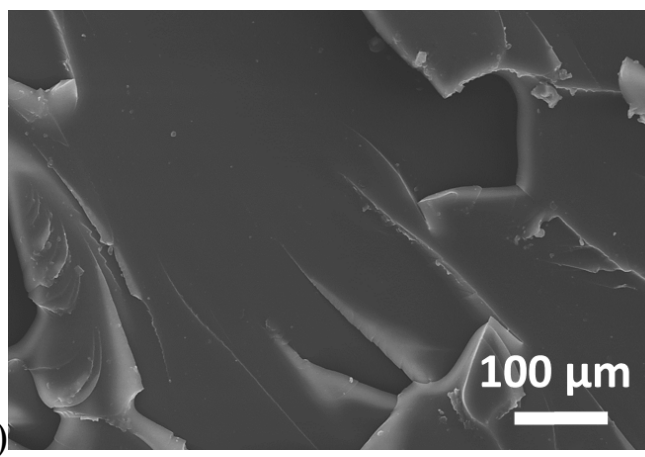

c)

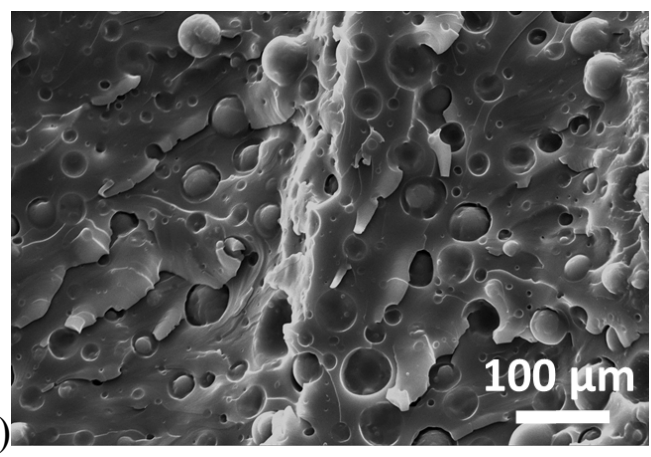

e)

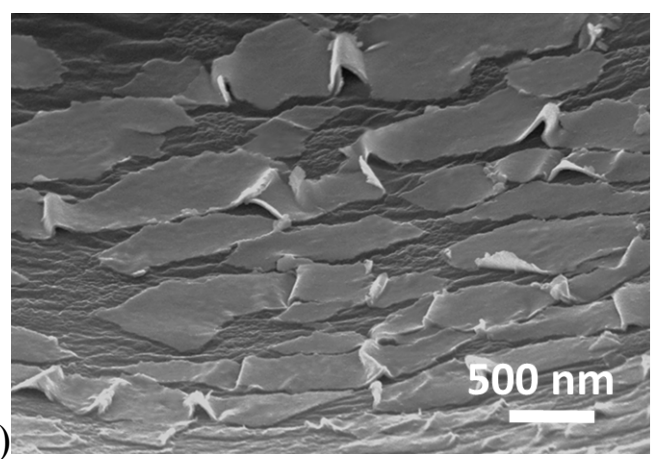

b)

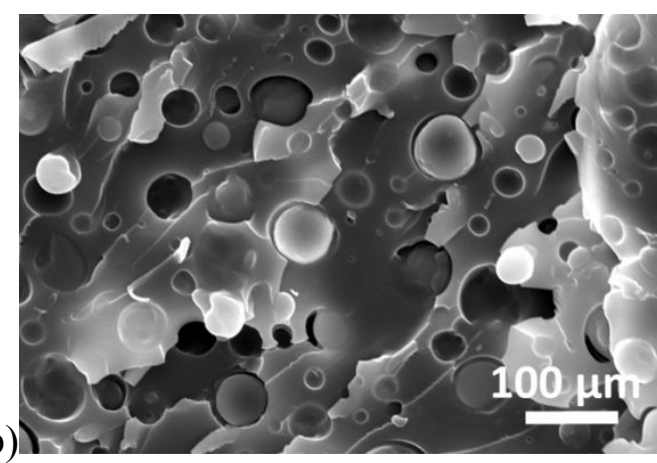

d)

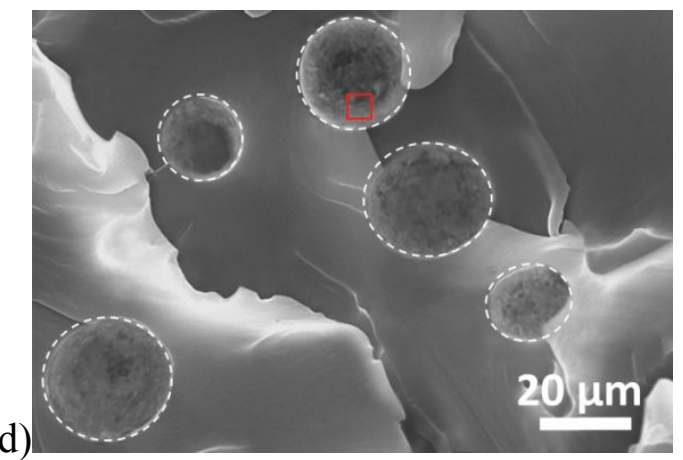

Figure S9. Fractured surface morphology of the broken samples in the tensile test: a) the pure EP; b) the EP/10-LNBR blend; c) the EP/0.04-JN/10-LNBR blend; d) the EP/0.20-JN/10-LNBR blend and e) after selective dissolution of LNBR. 